\title{
Evolutionary Synthesis of Optimal Control Policies for Manufacturing Systems
}

\author{
B Porter \\ and \\ T Merzougui \\ Department of Industrial and Manufacturing Systems Engineering \\ The University of Hong Kong \\ Pokfulam Road \\ Hong Kong
}

\begin{abstract}
In this paper, evolution strategies are used to synthesise optimal control policies for manufacturing systems. The evolutionary procedure is illustrated by synthesising optimal control policies for a manufacturing system previously considered in the context of genetic synthesis. The performance of evolution strategies and that of genetic algorithms are compared in detail for this system, thus indicating that the evolutionary synthesis procedure is faster than the genetic synthesis procedure.
\end{abstract}

\section{INTRODUCTION}

The synthesis of optimal control policies for manufacturing systems is of fundamental importance in industrial automation. Indeed, it is imperative that such systems be controlled optimally in order to minimise manufacturing costs and to meet production schedules. However, the synthesis of optimal control policies for manufacturing systems is a non-trivial task in view of the need to satisfy hard constraints on both control variables and state variables. The presence of these constraints makes it necessary to use classical optimal control techniques such as dynamic programming [1] or the maximum principle [2]; but this frequently leads to conceptual or computational difficulties. It was therefore shown by Porter and Allaoui [3] [4] that genetic algorithms [5] provide a straightforward alternative technique for the synthesis of optimal control policies for manufacturing systems. In particular, it was shown [3] that this genetic synthesis procedure can deal readily with the hard constraints on both control variables and state variables simply by assigning zero Darwinian fitness to any control policy that fails to satisfy these constraints.

However, the evolutionary processes generated by the genetic synthesis procedure of Porter and Allaoui [3] [4] are rather slow in producing the required optimal control policies for manufacturing systems. In order to accelerate these processes, an alternative methodology is therefore presented in this paper for the evolutionary synthesis of such control policies. This alternative methodology involves the use of evolution strategies without recombination [6] rather than genetic algorithms [5]. Such evolution strategies involve mutation and Darwinian selection but not chromosomal crossover, and are therefore less computationally onerous than genetic algorithms. The resulting increased computational speed is especially important for the synthesis of optimal control policies for large-scale manufacturing systems. In this paper, the evolutionary procedure is illustrated by synthesising optimal control policies for the manufacturing system previously considered by Porter and Allaoui [3] [4] in the context of genetic synthesis. The performance of evolution strategies and that of genetic algorithms are compared in detail for this system, thus indicating that the evolutionary synthesis procedure is faster than the genetic synthesis procedure.

\section{EVOLUTIONARY SYNTHESIS PROCEDURE}

The manufacturing systems under investigation comprise $m$ machines (with $n$ associated buffers) and produce $\mathbf{p}$ part types. The dynamical behaviour of such systems is governed by linear differential equation of the forms [7]

$$
\dot{\mathrm{q}}(\mathrm{t})=\mathrm{A}_{1} \mathrm{u}(\mathrm{t})+\mathrm{A}_{2} \mathrm{i}(\mathrm{t})
$$

and

$$
\dot{\mathrm{x}}(\mathrm{t})=\mathrm{A}_{3} \mathrm{u}(\mathrm{t})-\mathrm{d}(\mathrm{t})
$$

for the buffer dynamics and for the production dynamics, respectively. In these equations, $q(t) \in R^{n}$ is the vector of buffer levels, $x(t) \in R^{p}$ is the vector of finished parts, $u(t) \in R^{n}$ is the vector of production rates, $i(t) \in R^{p}$ is the vector of part-release rates, and $d(t) \in R^{p}$ is the vector of part-demand rates. In addition, $A_{1} \in R^{n \times n}$ is the buffer routing matrix such that $A_{1} u(t)$ represents the flows of 
parts between buffers; $A_{2} \in R^{\text {nxp }}$ is the buffer loading matrix such that $A_{2} i(t)$ represents the arrivals of external parts at the buffers; and $A_{3} \in R^{p \times n}$ is the output matrix such that $A_{3} u(t)$ represents the flow of finished parts. Also, if $\tau_{j}$ is the processing time of parts in buffer $j$ and $B^{(k)}$ is the set of buffers for machine $k(k=1,2, . ., m)$, then the buffer production rates must evidently satisfy the capacity constraints

$$
\sum_{j \in B^{(k)}} \tau_{j} u_{j}(t) \leq 1 \quad(k=1,2, \ldots, m)
$$

In addition, the state and control vectors must satisfy the constraints

$$
\begin{aligned}
& q(t) \geq 0 \\
& u(t) \geq 0
\end{aligned}
$$

and

$$
\mathrm{i}(\mathrm{t}) \geq 0
$$

However, $x(t)$, the state vector of finished parts, is not required to satisfy such a non-negativity constraint (since there may be either a surplus or a backlog of finished parts).

The optimal control synthesis problem is to find, over some time period of duration $T$, the vectors $i(t) \in R^{P}$ and $u(t) \in R^{n}$ of part-release rates and buffer-production rates required to meet a specified vector $d(t) \in R^{p}$ of partdemand rates. More precisely, the objective is to choose the control vectors $i(t) \in R^{p}$ and $u(t) \in R^{n}$ so as to minimise the cost function

$$
\Gamma=\int_{0}^{T}\left[\lambda q(t)+\mu^{+} x^{+}(t)+\mu^{-} x^{-}(t)\right] d t
$$

where

$$
\mathrm{x}^{+}(\mathrm{t})=\max \{\mathrm{x}(\mathrm{t}), 0\} \in \mathrm{R}^{\mathrm{p}}
$$

is the parts surplus vector,

$$
x^{-}(t)=\max \{-x(t), 0\} \in R^{p}
$$

is the parts backlog vector, and $\lambda \in R^{1 \times n}, \mu^{-} \in R^{1 \times p}, \mu^{+} \in R^{1 \times p}$ are weighting vectors for the buffer contents and for the finished parts surplus or backlog. It is evident that the control vectors $i(t) \in R^{p}$ and $u(t) \in R^{n}$ that minimise this cost function, $\Gamma$, are optimal in the sense that the entire cost function associated with workin-progress (as measured by the buffer levels, production surplus, and production backlog) is minimised. However, the solution of this optimisation problem is non-trivial because the control vectors $i(t) \in R^{p}$ and $u(t) \in R^{n}$ must satisfy the constraints (3), (5), and (6) whilst the state vector $q(t) \in R^{n}$ must satisfy the constraint (4).

Nevertheless, procedures developed by Porter [8] in connection with the genetic design of control systems can be readily used to solve this optimal control synthesis problem for manufacturing systems. Thus, let the control interval $[0$, $\mathrm{T}]$ be divided into $\mathrm{N}$ sub-intervals, and let the elements of the vectors $i(t) \in R^{p}$ and $u(t) \in R^{n}$ be piecewise-constant functions on these sub-intervals. In this way, each element of $i(t) \in R^{p}$ can be represented on the control interval $[0, T]$ by an ordered set of $\mathrm{N}$ numbers so that the entire vector can be represented by an ordered set of $\mathrm{pN}$ numbers; and, similarly, each element of $u(t) \in R^{n}$ can be represented on the control interval $[0, T]$ by an ordered set of $N$ numbers so that the entire vector can be represented by an ordered set of $n N$ numbers. It follows that the entire control vector $\left[i^{T}(t), u^{T}(t)\right]^{T} \in R^{p+n}$ can be represented on the control interval $[0, T]$ by an ordered set of $(p+n) N$ numbers. Therefore, if each of these numbers is represented by a substring of binary digits, it is evident that the entire control vector $\left[i^{T}(t), u^{T}(t)\right]^{T} \in R^{p+n}$ can be represented on the control interval $[0, T]$ by the string of binary digits formed by concatenating these $(p+n) N$ sub-strings. The Darwinian fitness of each such string is normally given by

$$
\Phi=\frac{1}{\Gamma}
$$

where $\Gamma$ is the value of the cost function (7) associated with the entire control vector. However, if any of the constraints (3), (4), (5), and (6) are violated, the Darwinian fitness is then given by

$$
\Phi=0
$$

These procedures for encoding entire control vectors as binary strings, and for determining the Darwinian fitness of each such string, facilitate the use of evolution strategies in synthesising optimal control policies for manufacturing systems. The evolutionary design procedure begins by randomly generating an initial population of $\mu$ binary 
strings. These $\mu$ parental binary strings are then randomly mutated to form $\lambda$ binary strings representing offspring. Evolution then occurs according to a $(\mu+\lambda)$ strategy without recombination [6]. In this evolutionary process, the $\mu$ fittest binary strings representing the $\mu$ best control policies are selected from the entire population of $\mu$ parents plus $\lambda$ offspring to form the next generation of $\mu$ parental binary strings. This evolutionary process continues until no significant further increase is obtained in the fitness of the fittest binary string. This fittest binary string is then decoded, and thus yields the optimal entire control vector $\left[\mathrm{i}^{\mathrm{T}}(\mathrm{t}), \mathrm{u}^{\mathrm{T}}(\mathrm{t})\right]^{\mathrm{T}} \in \mathrm{R}^{\mathrm{p}+\mathrm{n}}$ which minimises the cost function, $\Gamma$, defined in equation (7) (whilst respecting the constraints expressed by the inequalities (3), (4), (5), and (6)). It is important to note that this evolutionary synthesis procedure is much simpler (and therefore much faster) than the genetic synthesis procedure of Porter and Allaoui [3] [4], since the latter involves chromosomal crossover as well as mutation.

\section{ILLUSTRATIVE EXAMPLE}

This general approach to the evolutionary synthesis of optimal control policies for manufacturing systems can be conveniently illustrated by considering a simple system in which $m=2, n=2$, and $p=1$. In this case, equation (1) for the buffer dynamics assumes the scalar form

$$
\dot{\mathrm{q}}_{1}(\mathrm{t})=\mathrm{i}(\mathrm{t})-\mathrm{u}_{1}(\mathrm{t})
$$

and

$$
\dot{\mathrm{q}}_{2}(\mathrm{t})=\mathrm{u}_{1}(\mathrm{t})-\mathrm{u}_{2}(\mathrm{t})
$$

whilst equation (2) for the production dynamics assumes the scalar form

$$
\dot{x}(t)=u_{2}(t)-d(t)
$$

It is assumed that $\tau_{1}=0.5$ and $\tau_{2}=0.5$ so that, in view of the inequalities (3), the production rates must satisfy the constraints

$$
\mathrm{u}_{1}(\mathrm{t}) \leq 2
$$

and

$$
u_{2}(t) \leq 2
$$

In addition, it follows from the inequalities (4), (5), and (6) that the state variables and control variables must satisfy the constraints

$$
\begin{aligned}
& \mathrm{q}_{1}(\mathrm{t}), \mathrm{q}_{2}(\mathrm{t}) \geq 0, \\
& \mathrm{u}_{1}(\mathrm{t}), \mathrm{u}_{2}(\mathrm{t}) \geq 0,
\end{aligned}
$$

and

$$
i(t) \geq 0
$$

The initial state of the system is assumed to be such that

$$
\begin{aligned}
& \mathbf{q}_{1}(0)=5 \\
& \mathrm{q}_{2}(0)=0 \\
& \text { and } \\
& x(0)=0
\end{aligned}
$$

It is further assumed that control is to be exercised on the time interval $[0,10]$, and that the part demand rate on this interval is

$$
d(t)=1 \quad(0 \leq t \leq 10)
$$

The objective of such control is to choose $i(t), u_{1}(t)$, and $\mathrm{u}_{2}(\mathrm{t})$ so as to minimise the cost function in equation (7) with $\mathrm{T}=10, \lambda=[5,10], \mu^{+}=\mu^{-}=5$, and $\mathrm{N}=20$.

The results of solving this optimal control problem using a $(100+100)$ evolution strategy without recombination [6] are shown in Figs 1 and 2 . In this case, a mutation probability $\pi_{\mathrm{m}}=0.02$ was used and evolution was allowed to continue over 1000 generations. In Fig 1(a), the full line shows the best-of-generation value of the cost function whilst, in Fig 1(b), the full line shows the associated generation - average value of the cost function: in each case, the dotted line indicates the corresponding values of the cost function in case genetic algorithms are used in the manner of Porter and Allaoui [3] [4] with the same mutation probability but with a crossover probability $\pi_{c}=0.6$. It is thus evident from Fig 1 that evolution occurs much more rapidly with the evolutionary synthesis procedure than with the genetic synthesis procedure. In the case of evolutionary synthesis, the state variables $q_{1}(t), q_{2}(t)$, and $x(t)$ of the manufacturing system behave as shown in Figs 2(a), 2(b), and $2(\mathrm{c})$, respectively, when the controls $i(t), u_{1}(t)$, and $u_{2}(t)$ shown in Figs $2(d), 2(e)$, and $2(f)$, respectively, are implemented. These controls give the optimal bufferproduction rates and the optimal part-release rate, and are 
obtained by decoding the best-of-generation string from the last generation of the evolutionary process. It is important to note that these controls obtained from the evolutionary synthesis procedure are very close to the following theoretically optimal values:

(a) $\mathrm{i}(\mathrm{t})=0, \quad 0 \leq \mathrm{t}<5 ; \quad \mathrm{i}(\mathrm{t})=1, \quad 5 \leq \mathrm{t} \leq 10$;

(b) $\mathrm{u}_{1}$ (t) $=1,0 \leq \mathrm{t} \leq 10$;

(c) $\mathrm{u}_{2}(\mathrm{t})=1, \quad 0 \leq \mathrm{t} \leq 10$;

The corresponding theoretically optimal value of the cost function is $\Gamma=68.75$.

\section{CONCLUSIONS}

In this paper, evolution strategies have been used to synthesise optimal control policies for manufacturing systems. It has been shown that the resulting evolutionary synthesis procedure is much faster than the genetic synthesis procedure [3] [4] previously used for such systems. Thus, even in the case of large-scale manufacturing systems, it is expected that the evolutionary procedure will provide an effective practical technique for the synthesis of optimal control policies. In this way, such manufacturing systems can be controlled optimally in order to minimise manufacturing costs and to meet production schedules.

\section{REFERENCES}

[1] S.B. Gershwin, "Manufacturing Systems Engineering", Prentice-Hall, 1994.
[2] J.B. Sousa and F.L. Pereira, "A receding horizon strategy for the hierarchical control of manufacturing systems", Proc. 4th International Conference on Computer Integrated Manufacturing and Automation Technology, pp 443-450, 1994.

[3] B. Porter and C. Allaoui, "Genetic synthesis of optimal control policies for manufacturing systems", Proc. 2nd World Automation Congress, pp 571-578, 1996.

[4] B. Porter and C. Allaoui, "Evolution of optimal control policies for manufacturing systems", Proc. IEEE International Conference on Industrial Technology, pp 305 $309,1996$.

[5] D.E. Goldberg, "Genetic Algorithms in Search, Optimization and Machine Learning", Addison-Wesley, 1989.

[6] I. Rechenberg, "Evolutionsstrategie: Optimienung Technischer Systeme nach Prinzipien der Biologischen Evolution", Frommann-Holzboog, 1973.

[7] A. Sharifnia, "Stability and performance of distributed production control methods based on continuous-flow models", IEEE Trans. Automatic Control, vol AC-39, pp 725-737, 1994.

[8] B. Porter, "Genetic design of control systems", J. SICE, vol 34, pp 393-402, 1995. 

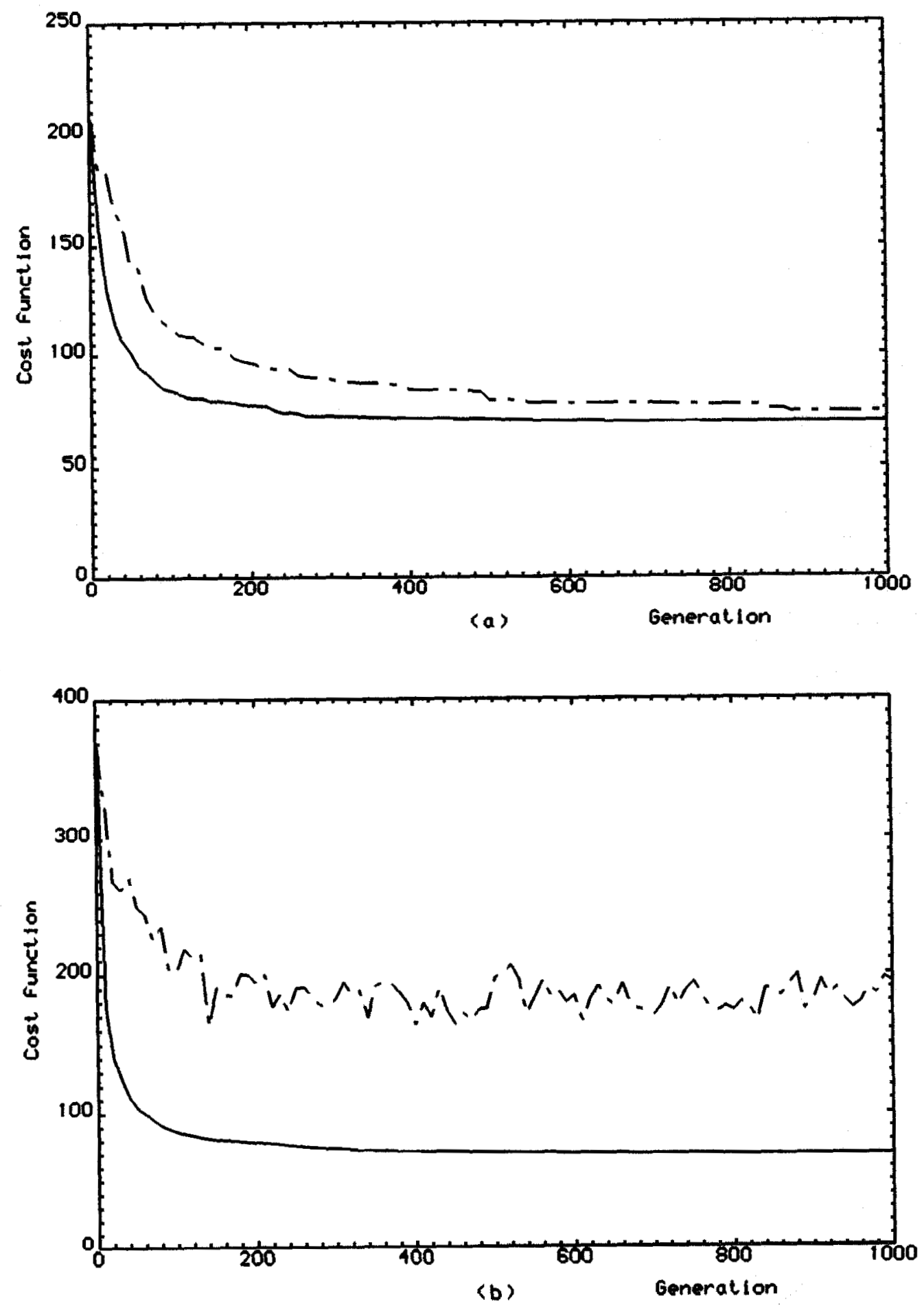

Figure 1: (a) Best-of-generation values of cost function.

(b) Generation-average values of cost function. 
91

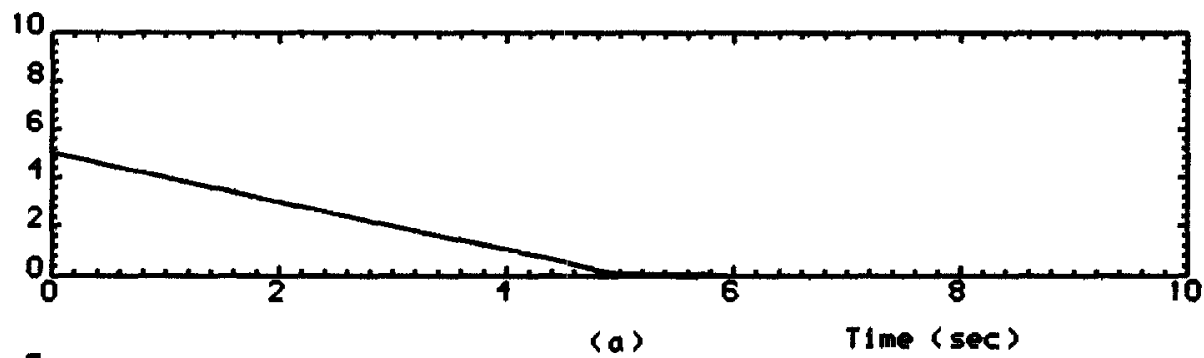

q2

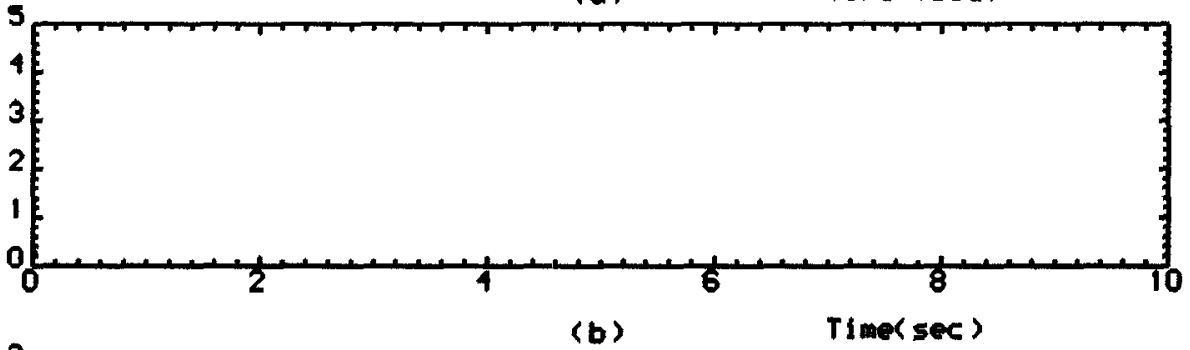

$\times$

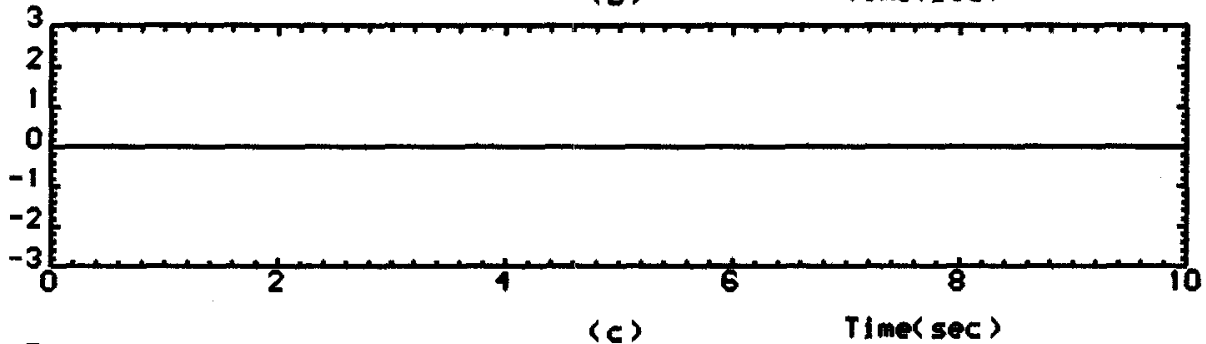

1

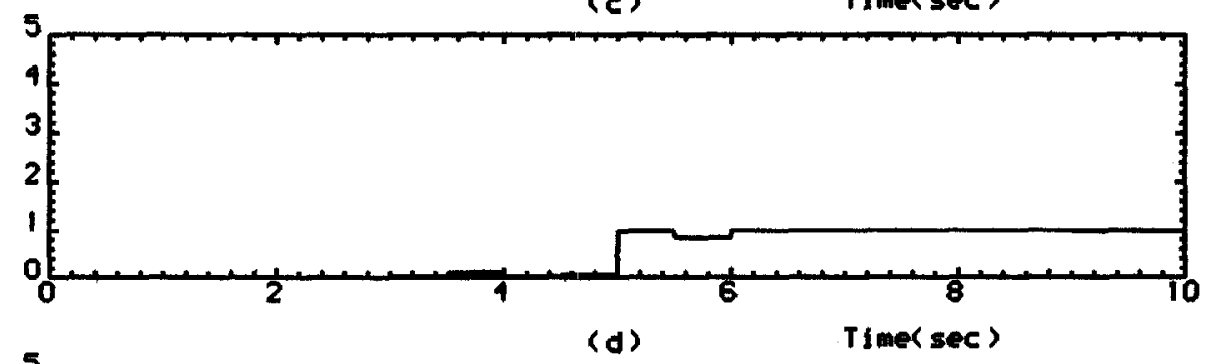

u!

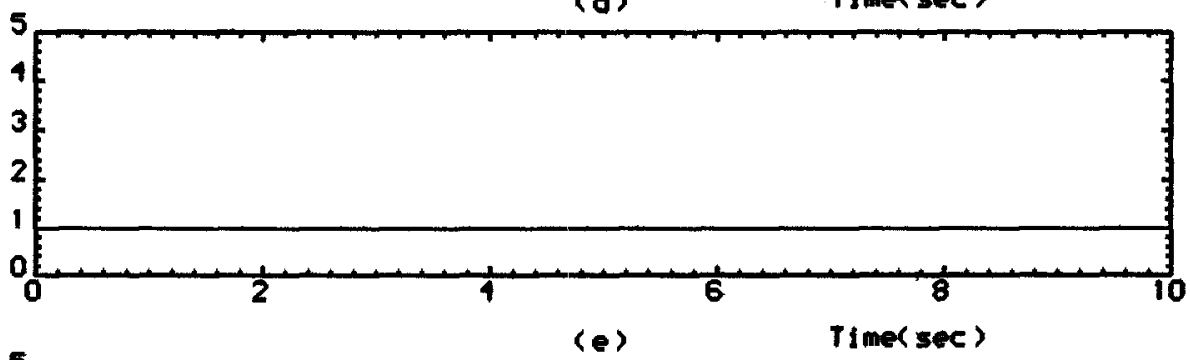

42

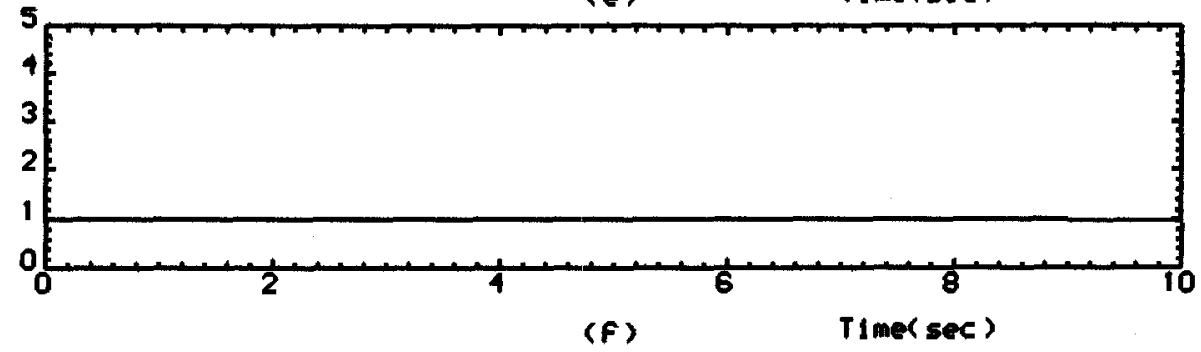

Figure 2: Time-domain behaviour of state and control variables 